\title{
Bronchoscopic drainage with full personal protective equipment (PPE) saved a life of COVID-19 infected patient with ventilator associated pneumonic athelectasis from the Diamond Princess Cruise Ship
}

\author{
Yoshihiro Miyashita*1, Gaku Matsumoto ${ }^{2}$, Yumiko Kakizaki ${ }^{1}$, Toshiharu Tsutsui ${ }^{1}$, Junichi Inoue ${ }^{2}$, Masao Omata ${ }^{3,4}$ \\ ${ }^{1}$ Lung Cancer and Respiratory Disease Center, Fujimi, Kofu, Yamanashi, Japan \\ ${ }^{2}$ Department of Emergency and Critical Care Medicine, Fujimi, Kofu, Yamanashi, Japan \\ ${ }^{3}$ Genome Analysis Center, Yamanashi Central Hospital, Fujimi, Kofu, Yamanashi, Japan \\ ${ }^{4}$ The University of Tokyo, Hongo, Bunkyo, Tokyo, Japan
}

Received: September 18, 2020

DOI: $10.5430 /$ crim.v7n4p1
Accepted: October 26, 2020

Online Published: November 9, 2020

\begin{abstract}
A 75 year-old American passenger was transferred to our hospital, infected in Diamond Princess, with pneumonia due to COVID-19 infection. At the beginning, he walked in but on day 4, he needed intubation and mechanical ventilation support because of worsening respiratory function due to wide-spread pneumonia. Despite the ventilation, his respiratory condition did not improve due to his left lower lobe atelectasis by an obstruction of left main bronchus. On day 9, interventional drainage of the major bronchus by disposable bronchoscopy was performed and built-up cast was removed by suctioning under the endoscopy. After the procedure, respiratory condition remarkably improved and he was extubated on day 14. COVID-19 infection with severe acute respiratory failure need mechanical ventilation support and if major airway obstruction was suspected, immediate interventional drainage may drastically change the patient clinical course and outcome.
\end{abstract}

Key Words: COVID-19, Diamond Princess, Drainage, Bronchoscopy

\section{INTRODUCTION}

COVID-19 pandemic infection has spreaded across the world and number of infected patients reached 10 million at the end of June, 2020. The first report from Wuhan (China) based on the diagnosed cases in the hospital gave us the figures of $5.0 \%, 2.3 \%$ and $1.4 \%$, to ICU, with invasive mechanical ventilation and deceased, respectively. ${ }^{[1]}$

Anti-viral drugs including anti-HIV drugs, Remdesivir and
Fabipiravil were studied under controlled-trials for COVID19 infection, ${ }^{[2-4]}$ but there is no established anti-viral treatment until now.

When acute respiratory failure due to COVID-19 pneumonia progressed, mechanical ventilation support is desperately needed. However, still in many places, mechanical ventilation support are not available and could have complications such as ventilator associated pneumonia (VAP), airway me-

\footnotetext{
*Correspondence: Yoshihiro Miyashita; Email: y-miyashita@ych.pref.yamanashi.jp; Address: Yamanashi Central Hospital 1-1-1 Fujimi, Kofu, Yamanashi, Japan. 
chanical obstruction, and subsequent atelectasis.

We experienced a case of COVID-19 infected patient from cruise ship (Diamond Princess) with left main airway ob- struction during the ventilation. He was successfully treated by a single-use bronchoscopy with full personal protective equipment (PPE).

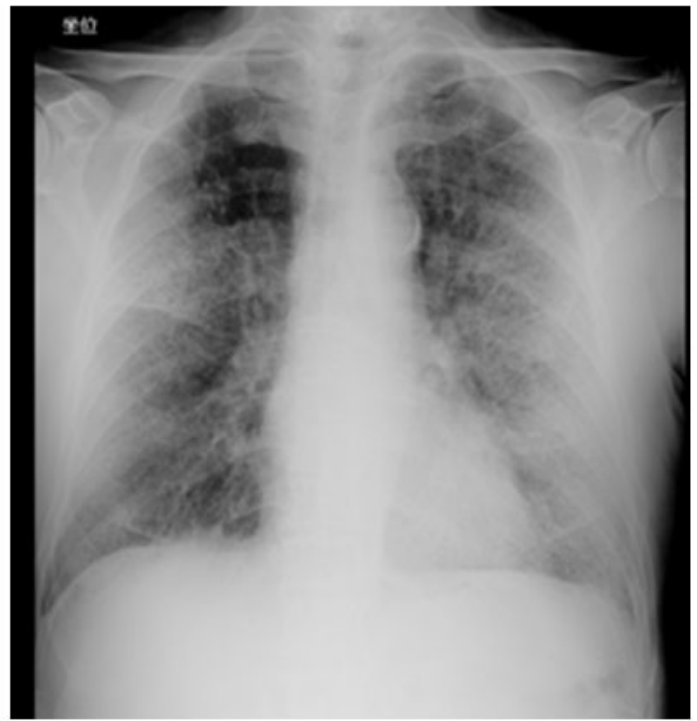

(a)

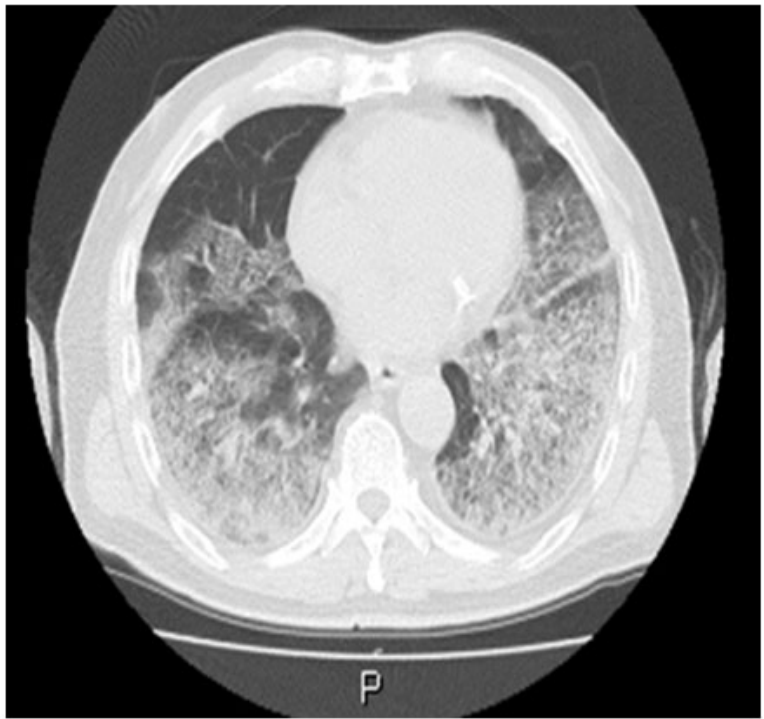

(b)

Figure 1. Chest radiograph (a) and computed tomographic (CT) picture (b) before intubation. Ground glass shadows were seen at outer area from middle to bottom of both lung fields (a). CT also revealed ground glass shadows at outer area of both lung fields without cardiomegaly (b).

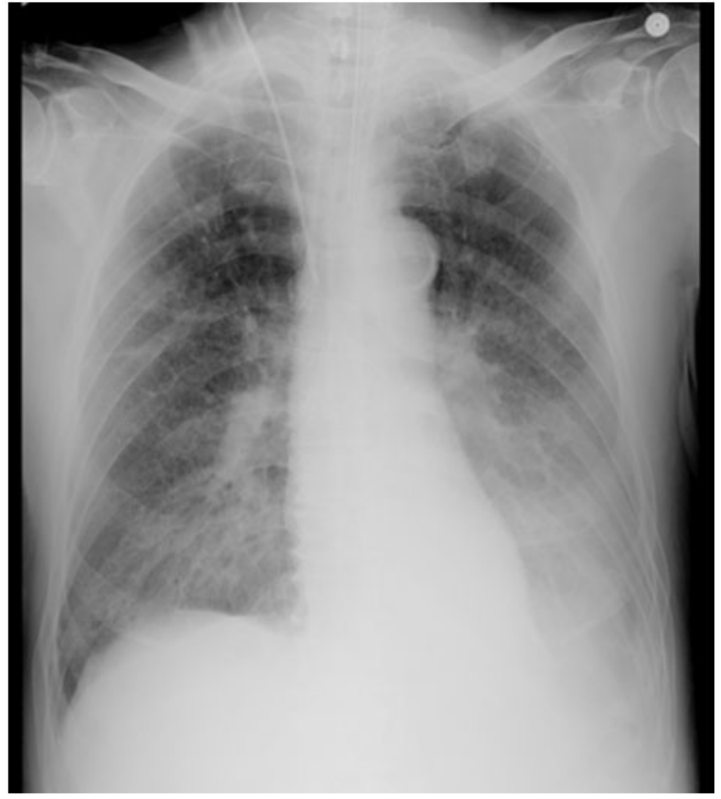

(a)

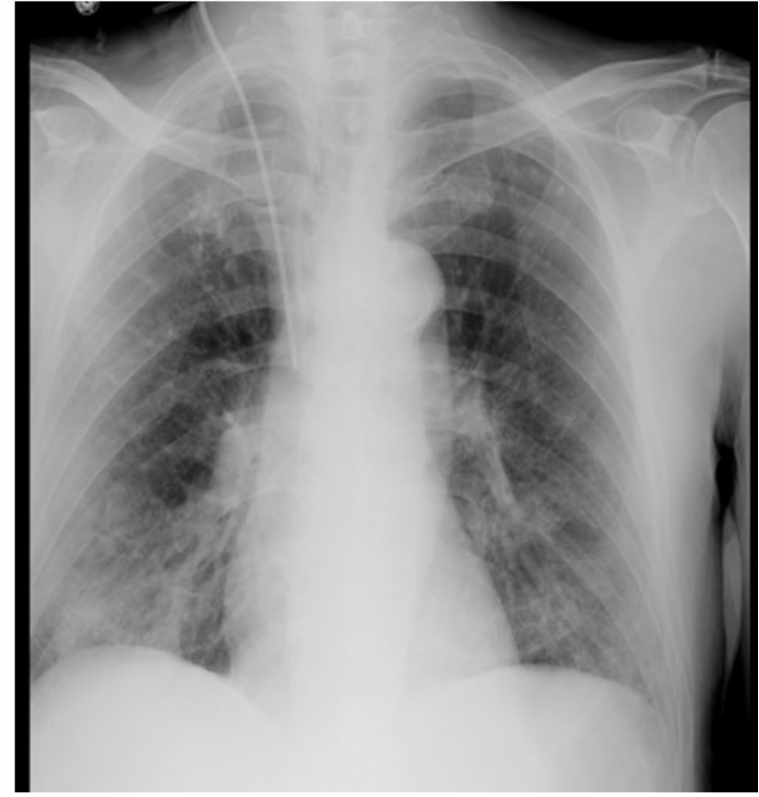

(b)

Figure 2. Chest radiograph before bronchoscopy (a) and after bronchoscopy (b). Chest radiograph before bronchoscopy revealed an atelectasis of left lower lung field. Silhouette sign of both discending aorta and left diaphragm were positive (a). Chest radiograph after bronchoscopy revealed improving of pneumonia and silhouette signs of both discending aorta and left diaphragm were negative. 


\section{CASE REPORT}

A 75 year-old male patient was transferred to our hospital due to COVID-19 infection in February, 2020. He was healthy except for gastroesophageal reflux with medication of protonpump inhibitor before admission to this hospital. He was ex-smoker but he had no respiratory symptom in his usual lifestyle. He became febrile during his travel on the Diamond Princess Cruise Ship, and he was diagnosed COVID-19 infection by RT-PCR examination of his nasopharyngeal swab at Yokohama quarantine station in Japan.

Actually, he walked in on admission. His chest XP showed ground-grass shadow at outer area from middle to bottom of both lungs (see Figure 1a). CT clearly revealed widespread ground-glass shadows at outer area of both lungs (see Figure 1b). There was no established medication for COVID19 infection at this time, lopinavir-ritonavir of an anti-HIV drug $^{[2]}$ was prescribed as off-label use with his informed consent. But during the following days, blood gas worsened and $\mathrm{PaO}_{2} / \mathrm{FiO}_{2}$ ratio (P/F ratio) reached 81.3 on day 4 . Therefore, he was intubated and mechanical ventilation support was started at ICU.

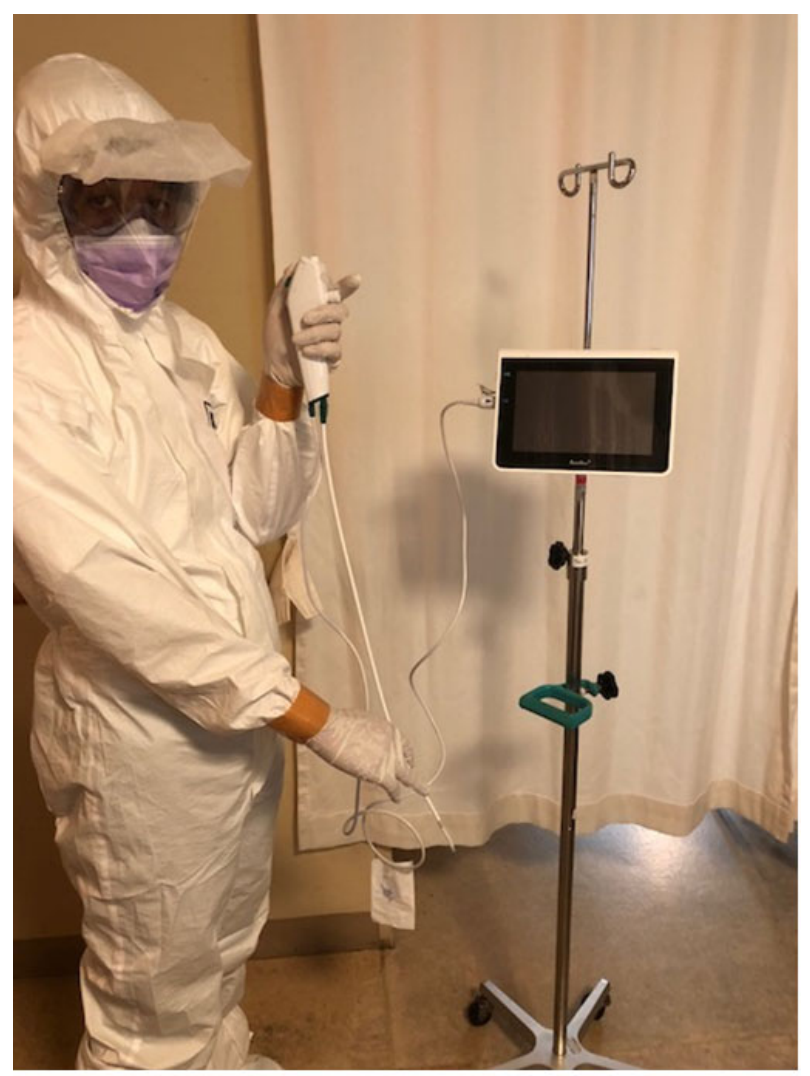

Figure 3. A single-use bronchoscopy with full personal protective equipments (PPE) including N95 mask, face shield, goggles, Tyvek suits gown and gloves.

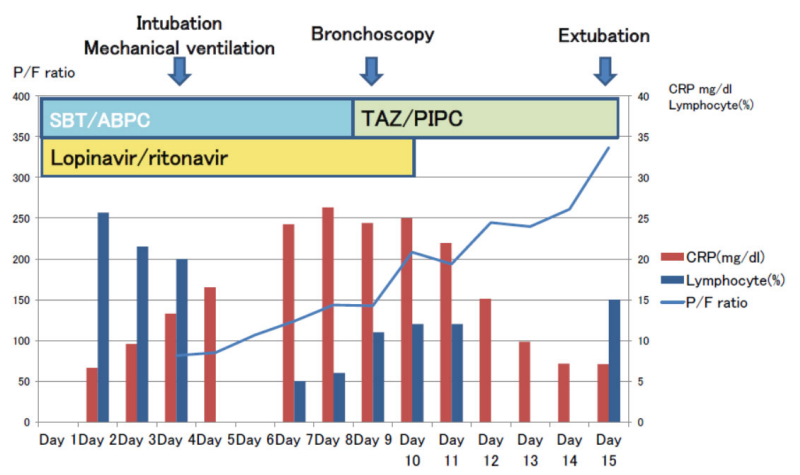

Figure 4. Clinical course during mechanical ventilation support

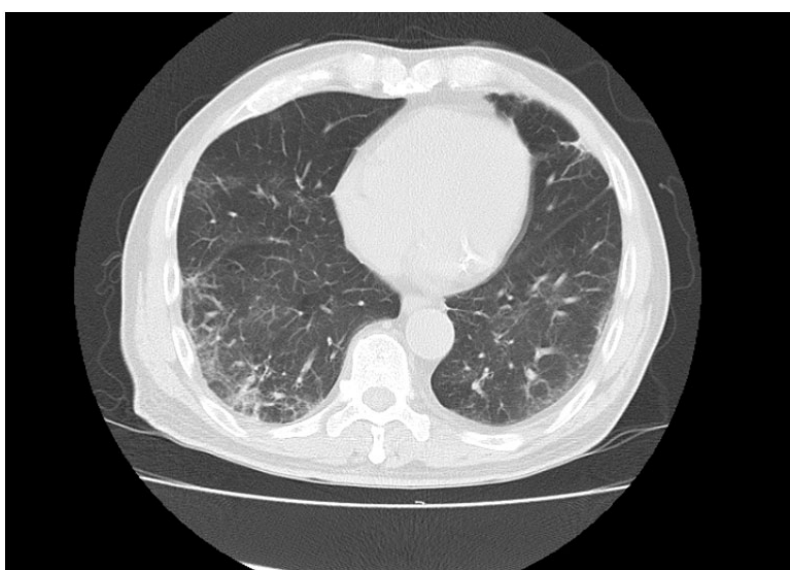

Figure 5. Chest CT findings on day 32 were dramatically improved with only small area of fibrotic changes in the bilateral lower back lung fields

From two days before intubation, he had several episodes of hemoptysis. A complication of alveolar hemorrhage was suspected due to membrane damage. By repeated CTs on the day of intubation, ground-glass shadows enlarged and became dense (see Figure 1b). In the meantime, Klebsiella pneumoniae and ordinary coronavirus strain were cultured from sputum. An antibiotics of Sulbactam/Ampicillin (SBT/AMPC) was changed to Tazobactam/Piperacillin (TAZ/PIPC) for treatment to Klebsiella pneumonia. It became difficult to suction the sputum out despite the effort of changing body positions. Left lower lobe of the lung became totally atelectatic and major airway obstruction was highly suspected (see Figure 2a).

On day 9 (5 days after intubation), to treat a suspected obstruction of left main bronchus, we conducted bronchoscopic drainage of left lobe with full personal protective equipment (PPE) (see Figure 3). His left main bronchus was totally obstructed by built-up cast which was removed by endoscopy and airway drainage was completed. Little before but after the drainage, abundant sputum came out. Radiological find- 
ings improved drastically after the procedure (see Figure 2b). Laboratory findings including $\mathrm{P} / \mathrm{F}$ ratio, lymphocytopenia and elevated CRP improved after the procedure (see Figure 4). His mechanical ventilation support was not needed on day 15, 6 days after bronchoscopic drainage and he was transferred to a general ward of respiratory department on day 21 . His chest CT findings on day 32 were dramatically improved with small area of fibrotic changes in the bilateral lower back lung fields (see Figure 5).

On day 22, the discharge criteria with two consecutive RTPCR negative results of nasopharyngeal swab was satisfied. But his wife was also transferred to this hospital due to COVID-19 infection 4 days later of his admission. Because of her cerebral hemorrhagic infarction with the operations of craniectomy and cranioplasty during her hospitalization, he and his wife returned to America together after her rehabilitation in April, 2020.

\section{Discussion}

Healthcare workers participating in aerosol-generating procedures such as endotracheal intubation, tracheotomy, bronchoscopy or otolaryngological procedures involving the upper airways are at risk for aerosol-transmitted infection.

Bronchoscopy is a useful diagnostic tool and sometimes needed at emergency but more careful precautions are necessary when applied to COVID-19 infection. Environmental contamination by aerosol spread of the COVID-19 virus is a critical matter during the procedure of bronchoscopy. Recent guidelines to conduct emergent bronchoscopy for the COVID-19 infected include lober or entire lung atelectasis due to mucous plugging. ${ }^{[5]}$

Considering for the above mentioned risks and indications of bronchoscopy, we decided to conduct interventional bronchoscopic examination to disclose the responsible lesion for suspected major airway obstruction and treat subsequent left lobe atelectasis with full PPE protection.

We found built-up cast which we were able to retrieve and eventually drained the major bronchus. The patient expelled huge amount of sputum after drainage and then condition remarkably improved. This plaque might be the reason why the patient cannot expel the sputum and atelectasis was ensued.

Two underlying mechanisms could be involved in the formation of the cast. Klebsiella pneumoniae was cultured during ventilation, therefore the bacteria infection could have contributed to solidify mucus plaque. Removal the built-up cast could have facilitated the resolution of atelectasis and the effect of antibiotics changing from SBT/AMPC to TAZ/PIPC.

Secondary, alveolar hemorrhage could contribute the viscid mucus. Episodes of hemoptysis was noted several times after ventilation started. Pathological findings of 21 COVID-19 patients revealed exudative diffuse alveolar damage with massive capillary congestion often accompanied by microthrombi and debris due to alveolar membrane damage by COVID-19. ${ }^{[6]}$ This hemorrhage might be a trigger for worsening and accelerated respiratory failure by forming the plaque. All these combined, the patient could have reached the critical condition. Clinically, bronchoscopic drainage turned the situation around.

\section{Conclusion}

We herein report a COVID-19 infected case with ventilator associated pneumonic atelectasis due to left main bronchus obstruction and successfully treated by an intervention of single-use bronchoscopy. A single-use bronchoscopy is safely manageable and could be reminded when COVID19 infected patients developed severe respiratory failure due to suspected major bronchial obstruction.

\section{CONFlicts OF INTEREST Disclosure}

The authors have declared no conflicts of interest.

\section{REFERENCES}

[1] Guan W, Ni Z, Hu Y, et al. Clinical Characteristics of Coronavirus Disease 2019 in China. N Engl J Med. 2020; 382: 1708-1720.

[2] Cao B, Wang Y, Wen D, et al. A Trial of Lopinavir-Ritonavir in Adults Hospitalized with Severe Covid-19. N Engl J Med. 2020; 382:1787-1799. PMid:32187464. https://doi.org/10.1056/NE JMoa2001282

[3] Grein J, Ohmagari N, Shin D, et al. Compassionate Use of Remdesivir for Patients with Severe Covid-19. N Engl J Med. 2020; 382(24): 1-10. PMid:32275812. https ://doi.org/10.1056/NEJMoa2007 016

[4] Cai Q, Yang M, Liu D, et al. Experimental Treatment with Favipiravir for COVID-19: An Open-Label Control Study. Engineering (Beijing). 2020 March. https ://doi.org/10.1016/j .eng.2020.03.007

[5] Lentz RJ, Colt H. Summarizing societal guidelines regarding bronchoscopy during the COVID-19 pandemic. Respirology.2020 June; 25(6): 574-577. PMid:32277733. https : //doi.org/10.29245/2 $572.942 \mathrm{X} / 2016 / 4.1040$

[6] Menter T, Haslbauer JD, Nienhold R, et al. Post-morten Examination of COVID19 Patients Reveals Diffuse Alveolar Damage With Severe Capilllary Congestion and Variegated Findings of Lungs and Othed Organs Suggesting Vascular Dysfunction. Histopathology. 2020; 77(2): 198-209. PMid:32364264. https ://doi.org/10.1 $111 /$ his. 14134 